\title{
FINITE LINEAR GROUPS IN SEVEN VARIABLES ${ }^{1}$
}

\author{
BY DAVID B. WALES
}

Communicated by Walter Feit, August 22, 1967

If $G$ is a finite group which has a faithful complex representation of degree $n$ it is said to be a linear group in $n$ variables. This is equivalent to saying $G$ is a finite group of complex linear transformations. It is customary to consider only unimodular linear transformations. For $n \leqq 4$ these groups have been known for a long time. An account may be found in Blichfeldt's book [1]. For $n=5$ they were determined by $R$. Brauer in [2]. Results in [2] are used to prove the following theorem for $n=7$.

ThEOREM 1. If $G$ has a complex irreducible representation of degree 7 which is faithful, unimodular, and primitive, then $G$ is one of the following groups. Here $Z(G)$ is the center of $G$.

I. $G$ is a uniquely determined group of order $7^{4} .48$ which has a normal subgroup $D$ of order $7^{3}, G / D \cong \mathrm{SL}(2,7)$. $D$ is nonabelian with exponent 1 .

II. Certain subgroups of $G$ in I of order $7^{3} \cdot s$ where $s \mid 48$. These contain $D$.

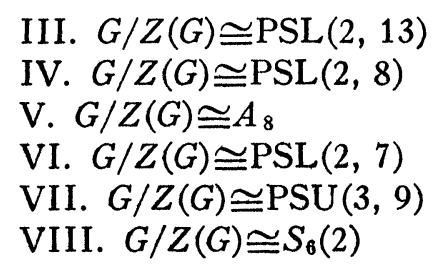

$$
\begin{aligned}
\mid G: Z(G) & =13 \cdot 7 \cdot 3 \cdot 2^{2} \\
G: Z(G) & =7 \cdot 3^{2} \cdot 2^{3}=504 \\
G: Z(G) & =8 ! / 2 \\
G: Z(G) & =7 \cdot 3 \cdot 2^{3}=168 \\
G: Z(G) & =7 \cdot 3^{3} \cdot 2^{5}=6048 \\
G: Z(G) & =7 \cdot 5 \cdot 3^{4} \cdot 2^{9}
\end{aligned}
$$

IX. $G / Z(G)$ is an extension of V, VI, VII by an automorphism of order 2 or an extension of IV by an automorphism of order 3 . For $\mathrm{V}$ it is $S_{8}$, for VI it is induced by PL $(2,7)$. For VII it is induced by a field automorphism and is $G_{2}(2)$. The extension of IV is induced by a field automorphism.

REMARKs. a. In the cases III-IX, $Z(G)$ has order 1 or 7 . If it has order 7 there is a subgroup $G_{1}$ such that $G \cong G_{1} \times Z(G)$.

b. A group satisfying all the hypotheses of Theorem 1 except for primitivity is a monomial group. In this case there is a normal abelian

${ }_{1}^{1}$ This work was the author's doctoral dissertation at Harvard University under the supervision of Professor R. Brauer. It was supported by the Canadian National Research Council. 
subgroup $K$ not in the center such that $G / K$ is a subgroup of the symmetric group on 7 elements $S_{7}$.

c. The proof of Theorem 1 is similar to [2] and will appear elsewhere.

\title{
BIBLIOGRAPHY
}

1. H. F. Blichfeldt, Finite collineation groups, Univ. of Chicago Press, Chicago, III., 1917.

2. R. Brauer, Über endliche lineare Gruppen von Primzahlgrad, Math. Ann. 169 (1967), 73-96.

\section{HARVARD UNIVERSITY}

\section{GRADED ALGEBRAS, ANTI-INVOLUTIONS, SIMPLE GROUPS AND SYMMETRIC SPACES}

\author{
BY C. T. C. WALL
}

Communicated by David A. Buchsbaum, August 25, 1967

We will outline two different generalisations of the Brauer group of a field (of characteristic $\neq 2$ ), and a third which combines them. By specialising to real and complex fields, we obtain algebra which describes the (classical) real Lie groups and symmetric spaces. The theory below can be generalised to arbitrary commutative rings; details will appear elsewhere.

We first recall the theory of the classical Brauer group of a field $k$ [2]. Consider simple finite-dimensional $k$-algebras with centre $k$. Any such algebra can be written as a matrix ring $M_{n}(D)$, where $D$ is a division ring with centre $k$. Write $M_{n}(D) \sim D$ : this induces an equivalence relation. The class of algebras is closed under tensor product. Multiplication is compatible with the equivalence relation, hence induces a product on the set of equivalence classes. For this product, the class of $k$ acts as unit, and taking the opposite algebra gives an inverse. We have an abelian group, $B(k)$.

We next define the graded Brauer group [3], $G B(k)$, of a field $k$ of characteristic $\neq 2$. Here consider $\left(Z_{2}^{-}\right)$graded $k$-algebras $A=A_{0} \oplus A_{1}$ of finite dimension, with no proper graded ideals, and such that the intersection of $A_{0}$ with the centre of $A$ is $k$ : we call these graded central simple algebras over $k$. We induce an equivalence relation by $A \sim A \otimes M_{n}(k)$, where $M_{n}(k)$ is graded by regarding it as the endomorphism ring of a graded vector space $k^{n}=V_{0}+V_{1}$, for some grading

1 To obtain an abstract simple group it suffices in most cases to take the quotient of the commutator subgroup of $G$ by its centre: see [5]. 